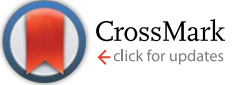

Cite this: RSC Adv., 2017, 7, 11528

Received 7th January 2017

Accepted 5th February 2017

DOI: $10.1039 / \mathrm{c} 7 \mathrm{ra00267j}$

rsc.li/rsc-advances

\title{
Facile construction of a polydopamine-based hydrophobic surface for protection of metals against corrosion $\uparrow$
}

\author{
Nan Wei, Yueyue Jiang, Ye Ying, ${ }^{*}$ Xiaoyu Guo, Yiping Wu, Ying Wen \\ and Haifeng Yang*
}

Metal surfaces with a hydrophobic feature, which could prevent percolation of water droplets and improve their resistance against corrosion, have attracted extensive interest. In this work, we demonstrated a method to prepare a hydrophobic copper surface by coating polydopamine (PDA), and modifying with 1-dodecanethiol (1-DT). Surface hydrophobicity was evaluated by contact angle analysis. Attenuated total reflectance Fourier transform infrared spectroscopy (ATR-FTIR), near infrared (NIR) spectroscopy and X-ray photoelectron spectroscopy (XPS) were employed to understand the structure and composition of the resultant coating at the metal surface. The inhibition efficiency of the optimal hydrophobic surface in $3 \mathrm{wt} \% \mathrm{NaCl}$ aqueous solution, explored by using electrochemical measurements, was $95.3 \%$, which was due to synergy effects of PDA and 1-DT. The promising inhibition of copper corrosion was also validated by scanning electron microscopy observation.

\section{Introduction}

Copper is an important metal for marine-related industries due to its mechanical workability, good antifouling, and good electrical and thermal conductivities as well as relatively low corrosion rate. ${ }^{1-5}$ However, the high salt environment of the sea also dramatically speeds up the corrosion of copper, which might result in serious problems with regards to safety. ${ }^{6}$ Benzotriazole and azole derivatives are effective organic inhibitors of copper corrosion in neutral-chloride aqueous solution, ${ }^{7,8}$ yet some of them are toxic, expensive, and not environmentally benign. As a wise alternative, the development of a hydrophobic surface against seawater invasion at the copper surface has attracted increasing attention. Conventionally in the literature, hydrophobic surfaces can be designed using two ideas, namely, rough microstructure and low surface energy. Many methods to construct hydrophobic surfaces, such as spray-deposition, ${ }^{9}$ electro-spinning, ${ }^{10}$ and nanocasting, ${ }^{11}$ have been investigated. Recently, several studies have focused on the on-site formation of hydrophobic films at the copper surface to realize corrosion inhibition. ${ }^{\mathbf{1 2 - 1 6}}$ Long hydrocarbon chain alkanethiol

The Education Ministry Key Lab of Resource Chemistry, Shanghai Key Laboratory of Rare Earth Functional Materials, Department of Chemistry, Shanghai Normal University, Shanghai 200234, PR China. E-mail: hfyang@shnu.edu.cn; yingye@ shnu.edu.cn; Fax: +86-02164322511

† Electronic supplementary information (ESI) available. See DOI: $10.1039 / \mathrm{c} 7 \mathrm{ra00267j}$ molecules, such as octadecanethiol, and 1-dodecanethiol (1DT), were also used to self-assemble hydrophobic films at a metal surface, showing an effective inhibition effect. ${ }^{17-20}$ However, they still suffer from instability after long periods of immersion in the harsh sea environment, because the long chain alkanethiol based protection films are normally a monolayer. Therefore, exploring a novel protocol for the construction of robust and hydrophobic layers at a metal surface is also necessary.

Dopamine molecules in aqueous solution in contact with a variety of solid substrates, including metals, oxides, polymers, semiconductors and ceramics, could spontaneously selfpolymerize. Under mild conditions, the formation of an adherent polydopamine (PDA) coating on a metallic surface is a simple and rapid process. ${ }^{21-24}$ In addition, in the case of an alkaline environment, dopamine can be easily oxidized to the quinone form by further cross-linking reactions. ${ }^{25-27}$ Unfortunately, cracks are easily found in the PDA coatings, because of their porous membrane-like nanostructures and hydrophilic functional groups. ${ }^{28}$ Such cracks in the coating will destroy the protective film due to the invasion of corrosive media.

Inspired by PDA coated surfaces, which can be further modified by thiol- or amine-containing molecules for biomaterial applications, ${ }^{\mathbf{2 9 , 3 0}}$ in this paper, taking advantage of the preferential adhesion of polydopamine at a surface and modifying it with hydrophobic 1-dodecanethiol, we described a facile approach to design a stable, compact and robust film with superior properties against corrosion in replica sea water of 3 $\mathrm{wt} \% \mathrm{NaCl}$ aqueous solution. 


\section{Experimental section}

\subsection{Materials}

Sulfuric acid (analytical grade reagent, AR), hydrochloric acid (AR), sodium chloride (AR), ethanol (AR), 1-dodecanethiol [HS $\left.\left(\mathrm{CH}_{2}\right)_{11} \mathrm{CH}_{3}\right]$ (98 wt\% purity), and dopamine hydrochloride (98 wt\% purity) were purchased from Sigma-Aldrich Corporation. All solutions were prepared with Milli-Q water $(18 \mathrm{M} \Omega \mathrm{cm})$.

\subsection{Pretreatment of the electrode}

The copper rod (99.999 wt\%) used in this experiment was inserted into a Teflon sheath with a geometric area of 0.0314 $\mathrm{cm}^{2}$. Prior to all experiments, the copper electrode was successively rubbed with 500- and 1000-grits papers to remove scratches, pits and the oxide layer, followed by $0.3 \mu \mathrm{m}$ alumina to polish the electrode until a shiny mirror-like surface was visible. And then the treated electrode was rinsed with Milli-Q water, pure ethanol and again with Milli$\mathrm{Q}$ water to entirely remove the alumina particles and loose copper rust.

\subsection{Surface modification}

After the above pretreatment, the copper electrode was immersed in Tris- $\mathrm{HCl}$ (pH 8.5) buffer with $0.5 \mathrm{mg} \mathrm{mL}^{-1}$ dopamine for $2 \mathrm{~h}$ and then in $5 \mathrm{mM}$ 1-DT solution for $1 \mathrm{~h}$ at room temperature $\left(25^{\circ} \mathrm{C}\right)$. Finally, the copper electrode was rinsed with Milli-Q water and then dried in air.

\subsection{Surface characterization}

2.4.1. Contact angle test. The surface contact angle was measured by using a commercial device (Powereach ${ }^{\circledR}$, JC2000D3, China). A $2 \mu \mathrm{L}$ droplet of Milli-Q water was used as the indicator.

2.4.2. X-ray photoelectron spectroscopy test. X-ray photoelectron spectroscopy (XPS, PHI 5000 VersaProbe, Japan) was used to identify the elementary composition at the copper surface.

2.4.3. ATR-FTIR and near infrared spectroscopy (NIR) test. An ATR-FTIR experiment was performed using a Fourier transform infrared spectroscope (Thermo Fisher Nicolet iS5, USA). NIR spectroscopy (Antaris II, Thermo Fisher Scientific, USA) was also used to observe the presence of DT in the PDA film on the metal surface.

2.4.4. Electrochemical experiment. The electrochemical behavior was determined in $3 \mathrm{wt} \% \mathrm{NaCl}$ aqueous solution by using a CHI750C electrochemical work-station (Shanghai $\mathrm{CH}$ Instruments, China) at room temperature. Electrochemical impedance spectroscopy (EIS) results were acquired under an open circuit potential of $0.005 \mathrm{~V}$ versus saturated calomel electrode (vs. SCE), an AC amplitude and a frequency ranging from $0.01 \mathrm{~Hz}$ to $100 \mathrm{kHz}$. The electrochemical polarization curves were recorded from -0.4 to $-0.05 \mathrm{~V}$ (vs. SCE) with a scan rate of $1 \mathrm{mV} \mathrm{s}^{-1}$.
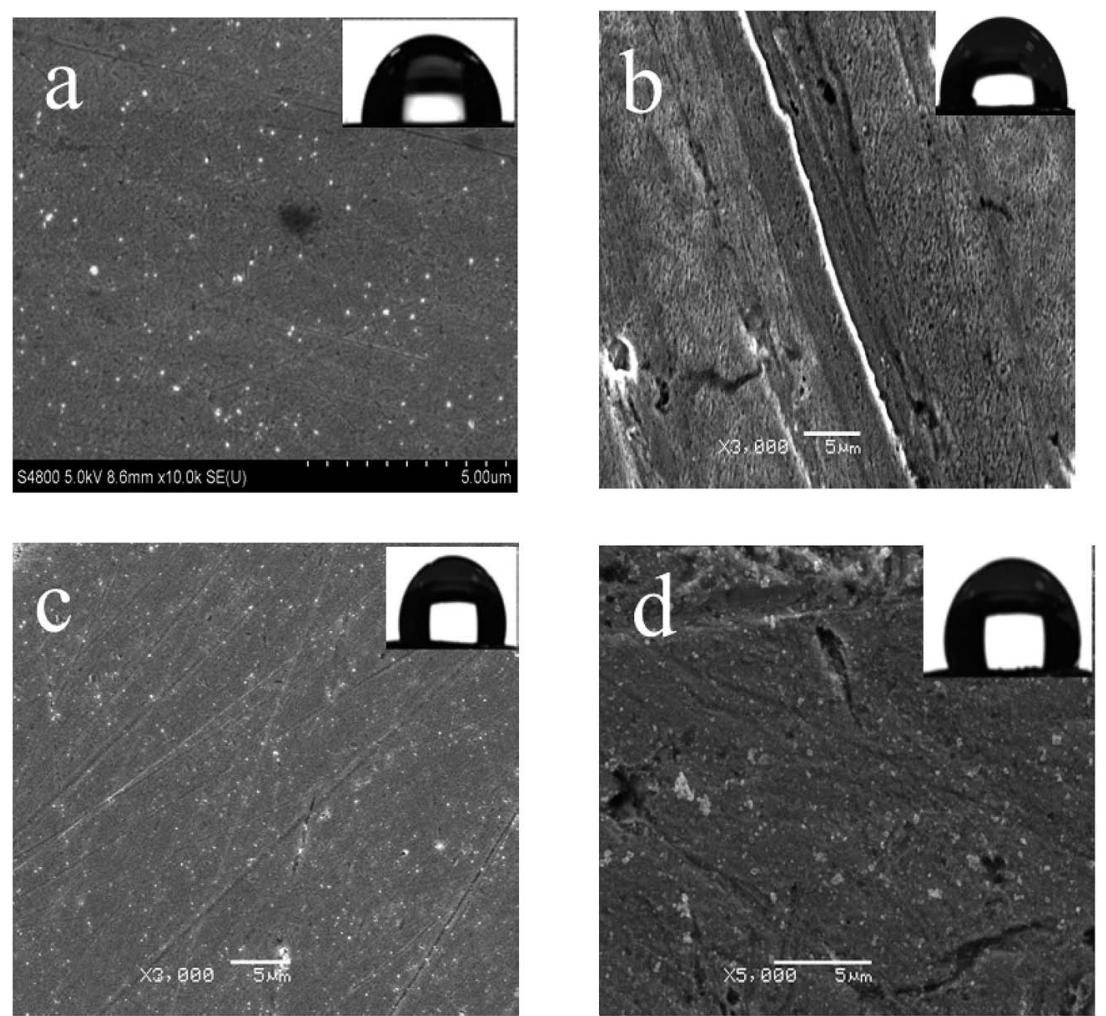

Fig. 1 SEM images of surfaces for (a) bare Cu, (b) PDA@Cu, (c) 1-DT@Cu, and (d) 1-DT@PDA@Cu (the insets are water contact angle images of the copper surfaces). 


\section{Results and discussion}

\subsection{Wettability of the copper surface}

The wettabilities of bare $\mathrm{Cu}$, PDA modified, 1-DT modified and 1-DT@PDA modified Cu surfaces, which were named PDA@Cu, 1-DT@ $\mathrm{Cu}$ and 1-DT@PDA@Cu in the following text, were surveyed. As shown in Fig. 1, the contact angle of the bare copper is only $87.2^{\circ}$. After modification with PDA, the contact angle increases to $90.5^{\circ}$. Finally, after modification with PDA and 1-DT successively, the copper surface becomes hydrophobic with a contact angle of approximately $110^{\circ}$.

\subsection{Composition of the hydrophobic surface}

XPS measurements were conducted to determine the composition at the copper surface. In Fig. 2a, with the scanning bonding energy range from 0 to $1000 \mathrm{eV}$, the basic elements, including carbon (285.0 eV), oxygen (531.8 eV), and nitrogen (398.4 eV), could be observed, proving the successful modification of the PDA film on the copper surface. In the case of 1DT@PDA@Cu, the XPS spectral survey also shows C 1s, N 1s and $\mathrm{O} 1 \mathrm{~s}$ peaks as the main indication of PDA, while in Fig. 2b, the presence of an S $2 \mathrm{p}$ peak at $163.7 \mathrm{eV}$ indicates that 1-DT molecules are successfully grafted on the surface. ${ }^{31}$ Simultaneously, referring to Table 1 , the atomic content calculated
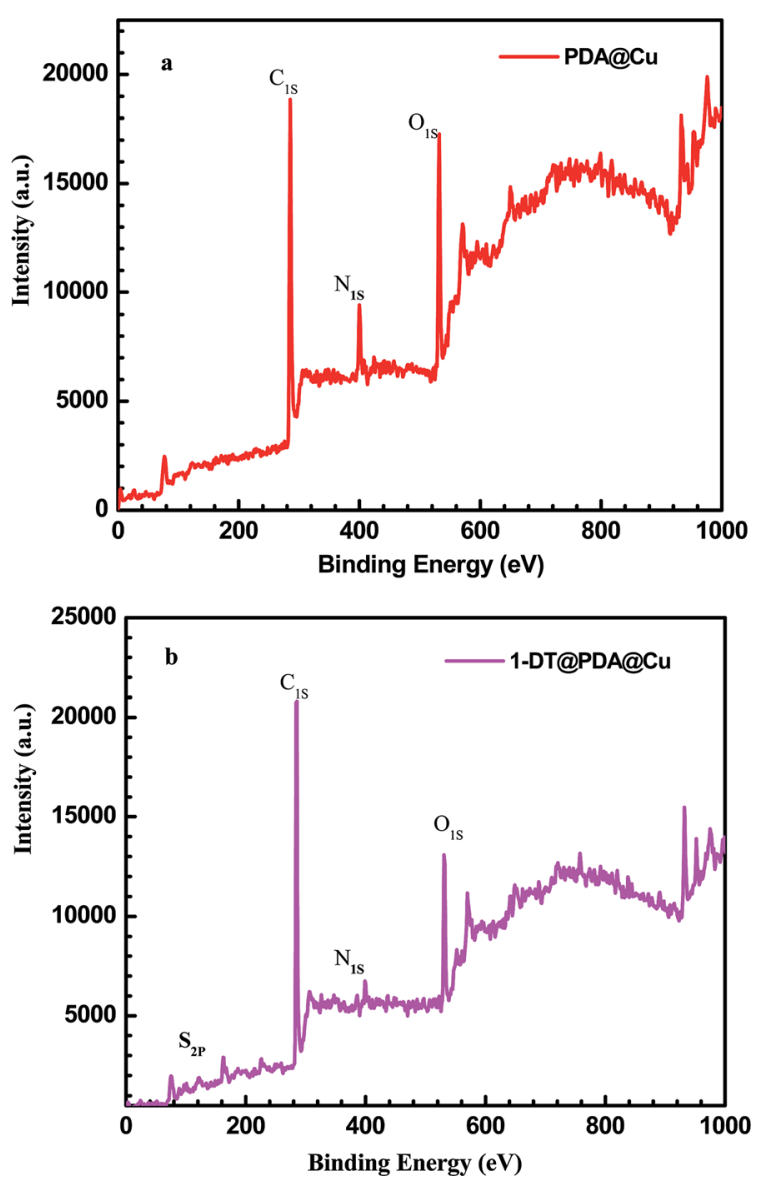

Fig. 2 XPS survey spectra of (a) PDA@Cu and (b) 1-DT@PDA@Cu.
Table 1 Atomic content calculated from the XPS survey spectra (Fig. 2)

\begin{tabular}{lllll}
\hline & \multicolumn{4}{l}{ Element content (at\%) } \\
\cline { 2 - 5 } Sample & $\mathrm{C}$ & $\mathrm{N}$ & $\mathrm{O}$ & $\mathrm{S}$ \\
\hline PDA@Cu & 73.35 & 7.20 & 19.45 & \\
1-DT@PDA@Cu & 79.75 & 3.45 & 14.31 & 2.49 \\
\hline
\end{tabular}

from XPS spectra shows that the $\mathrm{C}$ atom content increases after 1-DT modification. The content of $\mathrm{C}-\mathrm{C}$ bonds increases from $24.9 \%$ to $33.3 \%$ in careful comparison with PDA@Cu and 1DT@PDA@Cu in high resolution XPS results for C 1s, as shown in Fig. 3 and Table 2. For estimating the interaction behaviour of the 1-DT molecules in the resultant film, the corresponding XPS spectrum in the $S 2$ p region was also recorded. In Fig. 4 , the peaks centered at 162-163, around 164, and around $168 \mathrm{eV}$, correspond to thiolates, unbound thiols, and sulfinates, respectively. ${ }^{32,33}$ In this work, the 1-DT molecules are directly anchored to the copper surface via thiolates and unbound thiols of the PDA film. It should be mentioned that a sulfonic acid $\left(\mathrm{SO}_{3}{ }^{2-}\right)$ group (referring to $\mathrm{S}\left(2 \mathrm{p}_{3 / 2}\right)$ binding energy $\left.>166 \mathrm{eV}\right)^{34}$
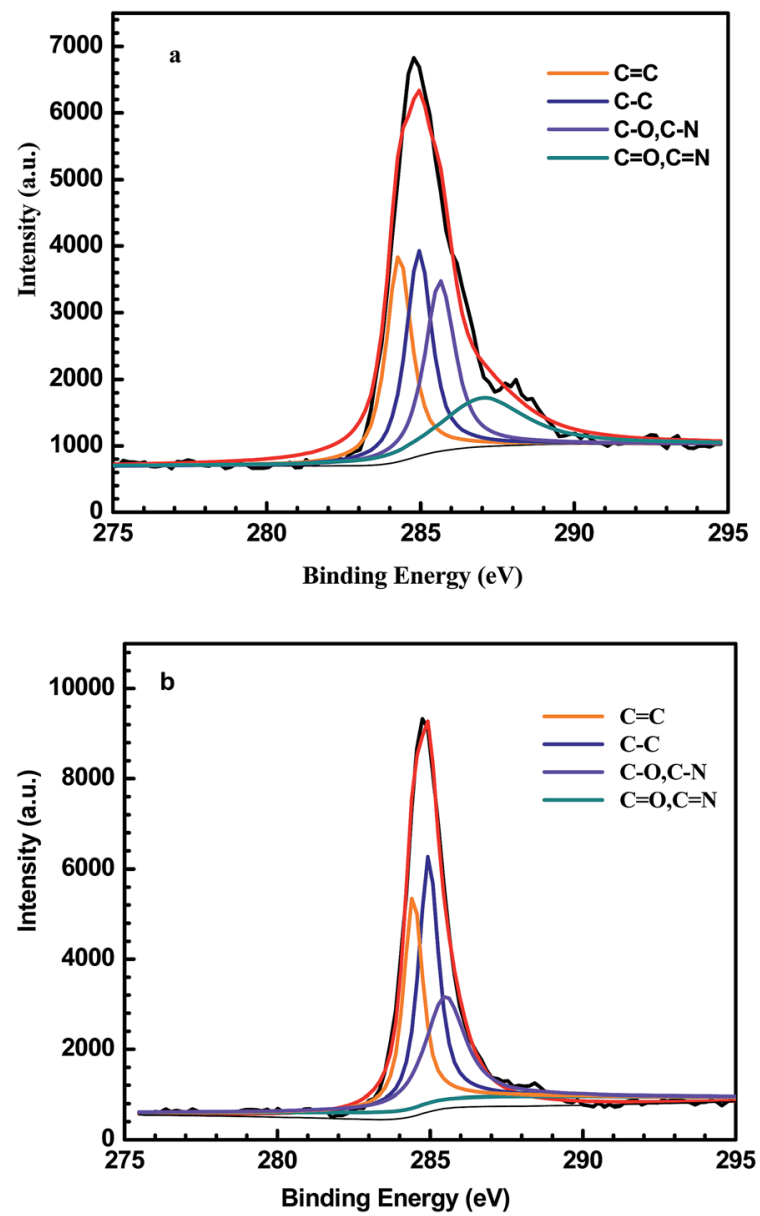

Fig. 3 C 1s XPS spectra of (a) PDA@Cu and (b) 1-DT@PDA@Cu with deconvoluted peaks. 
Table 2 Results of high resolution XPS spectra of $C$ 1s shown in Fig. 3

\begin{tabular}{lllll}
\hline & Bond content (at\%) & & & \\
\cline { 2 - 5 } Sample & $\mathrm{C}=\mathrm{C}(284.3 \mathrm{eV})$ & $\begin{array}{l}\mathrm{C}-\mathrm{C} \\
(284.8 \mathrm{eV})\end{array}$ & $\mathrm{C}-\mathrm{O}, \mathrm{C}-\mathrm{N}(285.6 \mathrm{eV})$ & $\mathrm{C}=\mathrm{O}, \mathrm{C}=\mathrm{N}(287.4 \mathrm{eV})$ \\
\hline PDA@Cu & 22.8 & 28.4 & 28.4 & 21.4 \\
1-DT@PDA@Cu & 33.3 & 33.3 & 33.3 & 0.01 \\
\hline
\end{tabular}

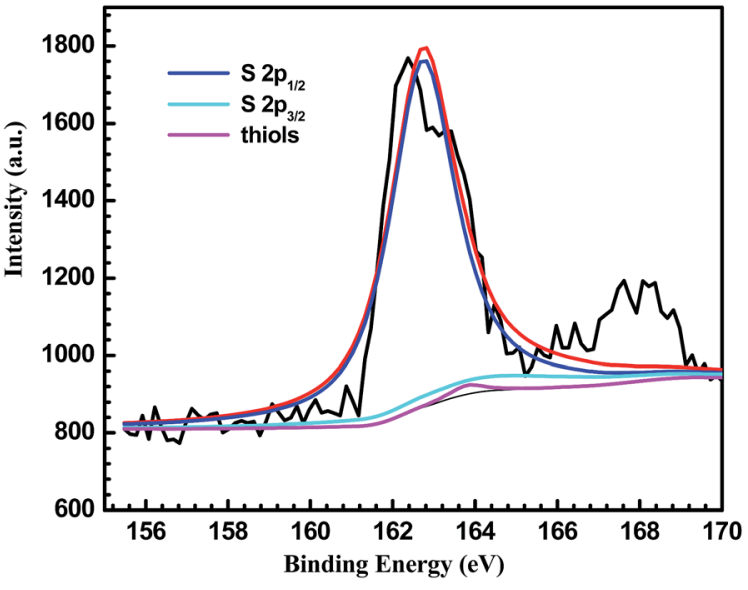

Fig. 4 S 2p XPS spectra of 1-DT@PDA@Cu with deconvoluted peaks.

was also found, which might be the oxidized sulfur species of 1DT. This consequently suggests that the 1-DT molecules were primarily attached to the copper surface by the formation of $\mathrm{Cu}-$ $\mathrm{S}$ bonds and side reactions involving hydrogen bonds or physical adsorption. ${ }^{35-37}$

ATR-FTIR spectra of the modified copper surfaces were acquired to obtain more in-depth interaction information of PAD or/and 1-DT with the copper surface. As shown in Fig. 5, after the modification of PDA, an FTIR band at $1613 \mathrm{~cm}^{-1}$ is ascribed to contributions from phenylic $\mathrm{C}=\mathrm{C}$ stretching and $\mathrm{N}-\mathrm{H}$ bending. An IR band centered at $1510 \mathrm{~cm}^{-1}$ is from $\mathrm{N}-\mathrm{H}$

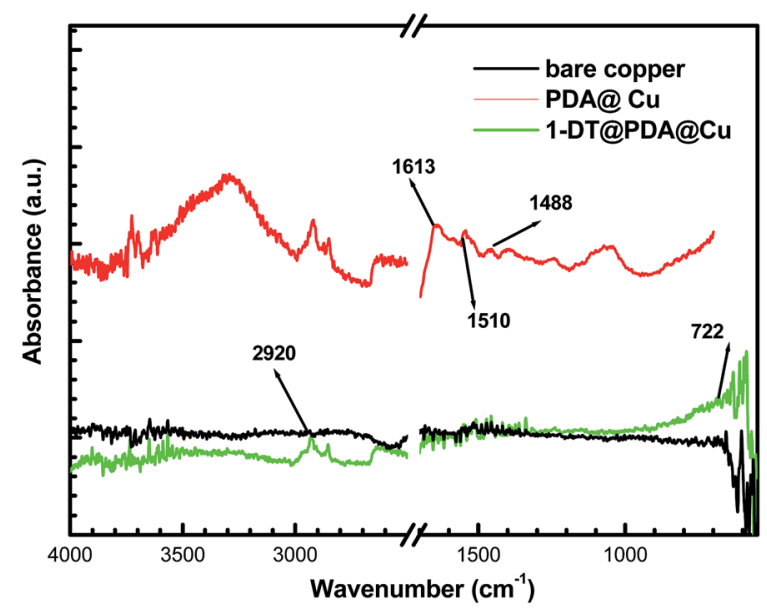

Fig. 5 ATR-FTIR spectra of different copper samples. scissoring. A peak at $1488 \mathrm{~cm}^{-1}$ is identified as C-N stretching. ${ }^{38,39}$ The relatively broad and strong band in the 3000-3400 $\mathrm{cm}^{-1}$ region should be due to the role of intermolecular hydrogen bonds existing in dopamine molecules. ${ }^{40}$ In the case of the ATR-FTIR spectrum of 1-DT@PDA@Cu, the absence of the S-H stretching band at $2550-2600 \mathrm{~cm}^{-1}$ indicates the chemisorption of 1-DT at the copper surface after cleavage of the S-H bond. ${ }^{41}$ Simultaneously, the $\mathrm{CH}_{2}$ asymmetrical stretching band of 1-DT could be observed at $2920 \mathrm{~cm}^{-1}$, and $\left(\mathrm{CH}_{2}\right)_{n}$ deforming appearing at $722 \mathrm{~cm}^{-1}$ is commonly used as an indicator of long alkyl chains.

NIR is a well-established analytical method for observing abundant overtone bands of hydrogen atom related stretching. To further prove the chemical modifications, we obtained the NIR spectra of pure 1-DT and different DT-modified copper samples. As shown in Fig. 6, for PDA@Cu, no clear peak appears
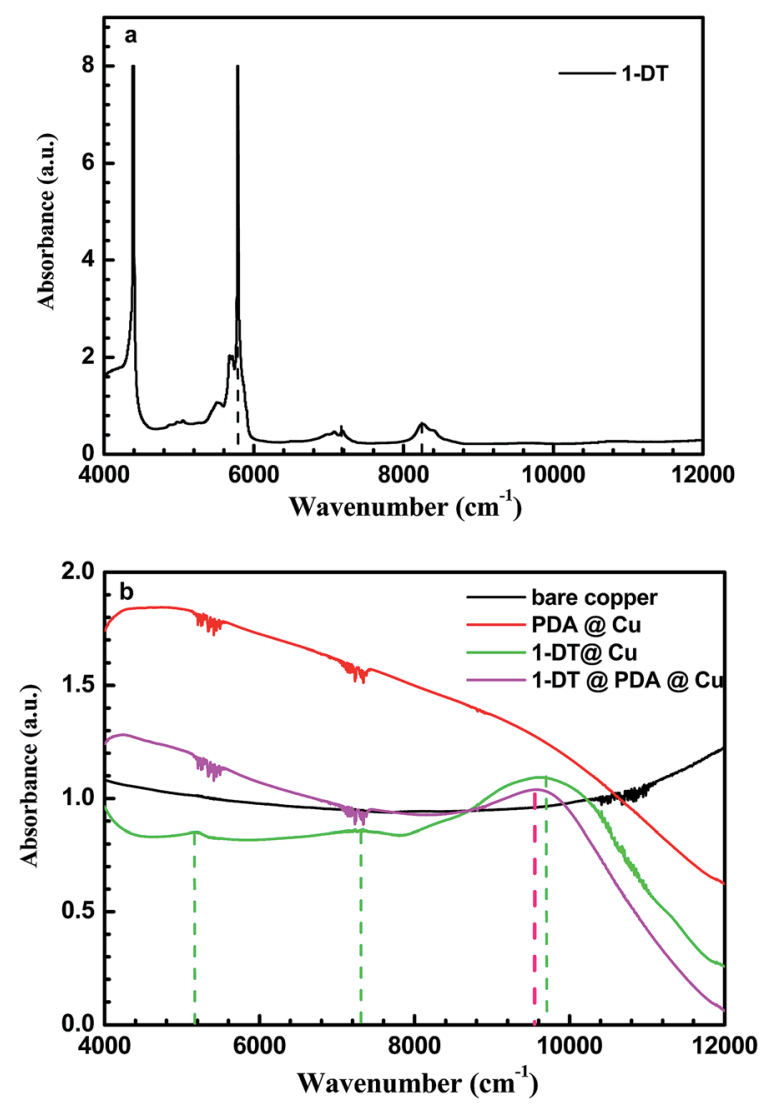

Fig. 6 (a) Near infrared spectrum of 1-DT molecules and (b) near infrared spectra of different copper samples. 


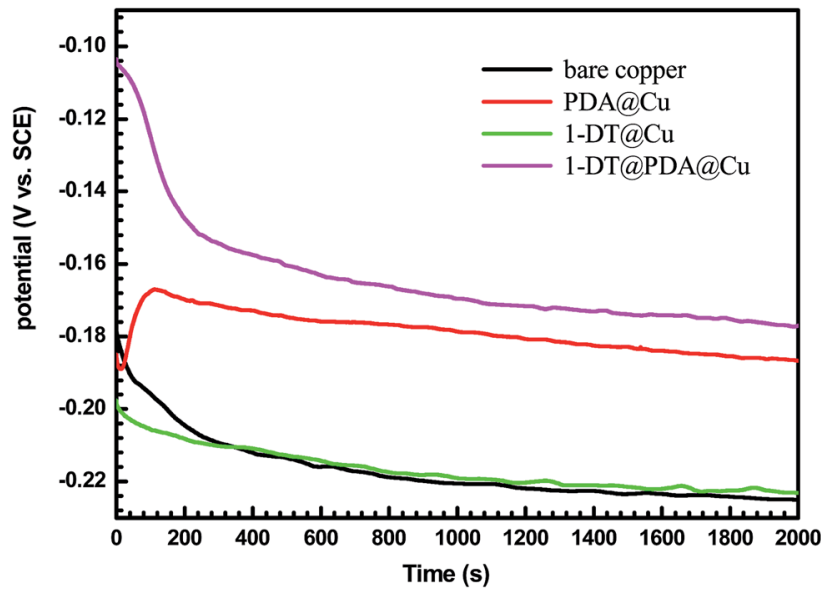

Fig. 7 Open circuit potential versus time of different copper samples recorded in $3 \mathrm{wt} \% \mathrm{NaCl}$ aqueous solution.

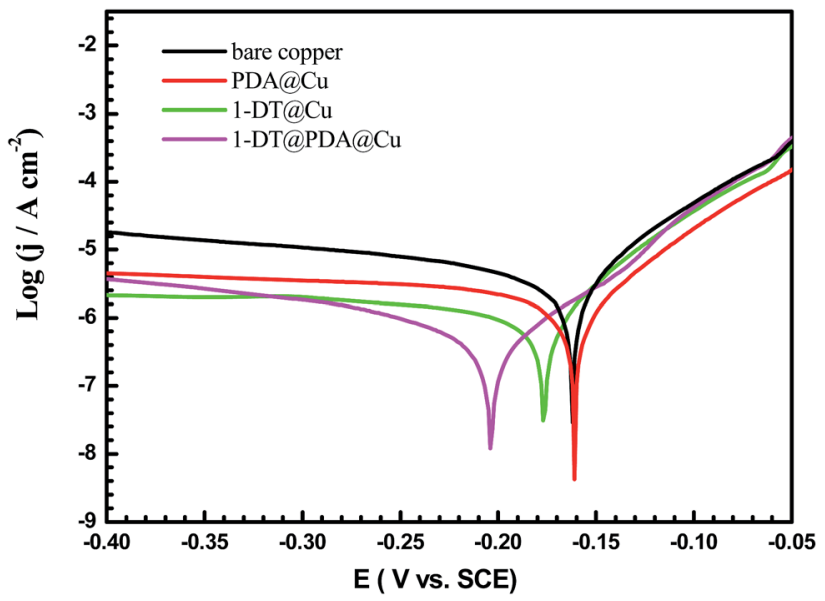

Fig. 8 Anodic and cathodic polarization curves of different copper samples recorded in $3 \mathrm{wt} \% \mathrm{NaCl}$ aqueous solution.

due to fewer residues of $\mathrm{CH}$ groups in polydopamine. In the case of 1-DT@Cu, three different peaks were visible, which should come from overtone stretching of $\mathrm{CH}$ long chains in DT. Comparably, NIR absorbance of $\mathrm{CH}$ long chains could be clearly seen in the spectrum of 1-DT@PDA@Cu, meaning the 1-DT molecules enter into the cracks of PDA and chemically modify the copper surface. The NIR result agrees with the above XPS investigation that the $\mathrm{SH}$ group in the DT molecule could

Table 3 Corrosion parameters obtained from potentiodynamic polarization curves for different samples in $3 \mathrm{wt} \% \mathrm{NaCl}$ aqueous solution

\begin{tabular}{lllll}
\hline & $\begin{array}{l}E_{\text {corr }}(\mathrm{V} \\
v s . \mathrm{SCE})\end{array}$ & $I_{\text {corr }}\left(\mu \mathrm{A} \mathrm{cm}^{-2}\right)$ & $\beta_{\mathrm{c}}\left(\mathrm{V} \mathrm{dec}^{-1}\right)$ & $\beta_{\mathrm{a}}\left(\mathrm{V} \mathrm{dec}^{-1}\right)$ \\
Sample & -0.162 & 4.72 & 1.51 & 20.03 \\
Bare & -0.161 & 2.19 & 1.52 & 17.22 \\
PDA@Cu & -0.177 & 1.57 & 2.19 & 16.99 \\
1-DT@Cu & -0.204 & 0.47 & 3.96 & 24.02
\end{tabular}

approach the copper surface and then remove a $\mathrm{H}$ atom for the formation of a $\mathrm{Cu}-\mathrm{S}$ bond.

\subsection{Open circuit potential}

Fig. 7 shows the open circuit potentials (OCP vs. $\mathrm{Ag} / \mathrm{AgCl}$ ) of bare copper, PDA modified, 1-DT modified and 1-DT@PDA modified $\mathrm{Cu}$ versus time. The stable OCP of copper is $-0.223 \mathrm{~V}$ vs. $\mathrm{Ag} / \mathrm{AgCl}$, while the OCP of 1-DT@PDA@Cu is approximately $0.168 \mathrm{~V}$. The latter is more positive than the former, implying that 1-DT@PDA@Cu can reduce the corrosion in $\mathrm{NaCl}$ aqueous
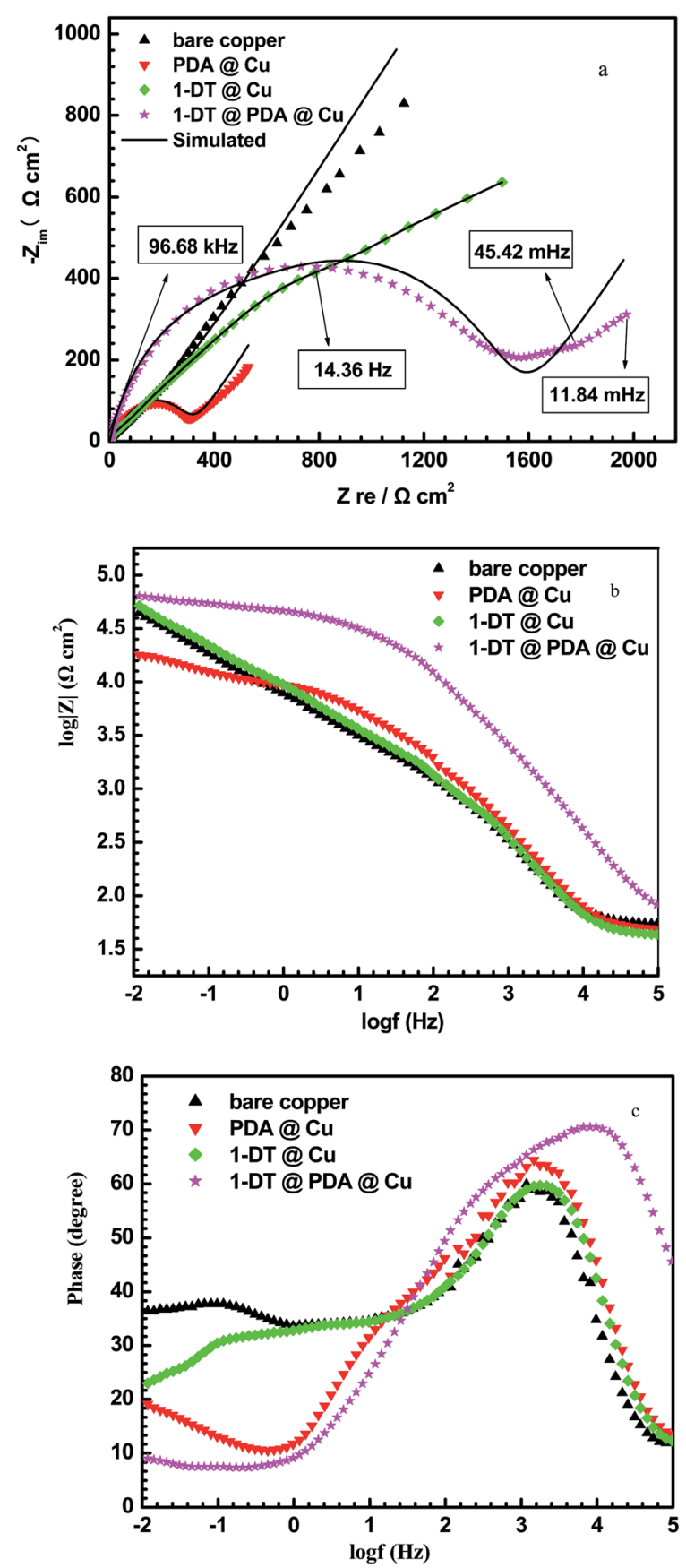

Fig. 9 Nyquist (a), Bode (b) and phase angle (c) plots for different copper samples recorded in 3 wt\% $\mathrm{NaCl}$ aqueous solution. 


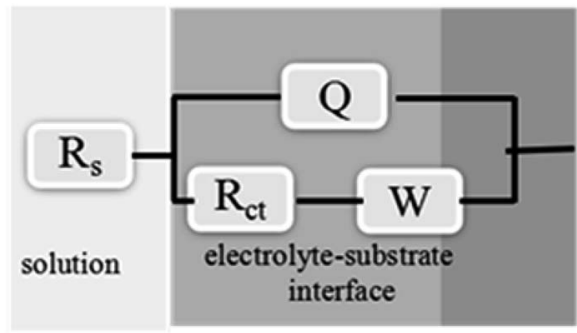

(a) $R(Q(R W))$

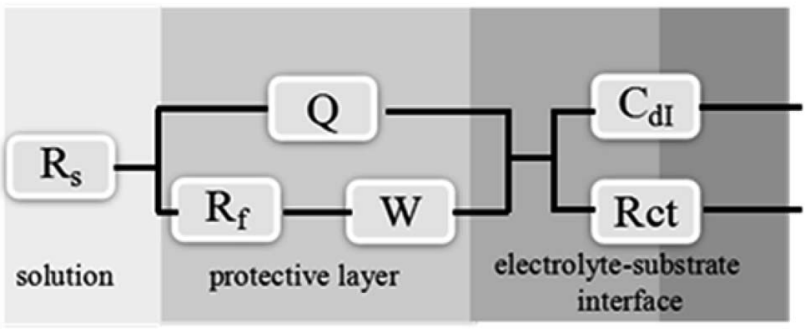

(b) $R(Q(R W))(C R)$

Fig. 10 Electrochemical equivalent circuits simulated for the impedance of the (a) bare copper and (b) modified copper.

solution. The following potentiodynamic polarization and EIS plots for different modified coppers were measured at the corresponding open circuit potentials.

\subsection{Electrochemical polarization studies}

Fundamentally, metal degradation is an electrochemical corrosion process, which can be estimated by electrochemical methods. ${ }^{42}$ Fig. 8 shows the potentiodynamic polarization curves of the bare $\mathrm{Cu}, \mathrm{PDA} @ \mathrm{Cu}, 1-\mathrm{DT} @ \mathrm{Cu}$ and 1-DT@PDA@Cu in $3 \mathrm{wt} \% \mathrm{NaCl}$ aqueous solution. Extrapolation of the Tafel line allows us to determine the corrosion current density ( $\left.I_{\text {corr }}\right)$ together with corrosion potential $\left(E_{\text {corr }}\right)$, anodic Tafel slopes $\left(\beta_{\mathrm{a}}\right)$, and cathodic Tafel slopes $\left(\beta_{\mathrm{c}}\right)$. These parameters are displayed in Table 3. With respect to the bare copper, all of the modified copper electrodes show lower current densities in both cathodic and anodic directions. Furthermore, the decrease of cathodic current densities for the modified coppers is very noticeable. Therefore, these coatings mainly act as a cathodic type inhibitor on the copper surface. In addition, comparing with PDA@Cu, the 1-DT@PDA@Cu surface presents a lower $I_{\text {corr }}$ value, which is attributed to 1-DT occupying and invading. the cracks of the PDA coating. The presence of 1-DT in PDA significantly increases the hydrophobicity of the $\mathrm{Cu}$ surface and favors the improvement of the corrosion resistance.

\subsection{Electrochemical impedance spectroscopy}

EIS measurements help to characterize the interface resistance behavior of the electrolyte/metal with films such as oxides, salts and adsorbed species, when the metal is exposed to the corrosive medium. ${ }^{43}$ Fig. 9a shows the Nyquist diagrams measured on the copper electrodes with and without a coating. Upon modification of PDA, 1-DT or 1-DT@PDA at the copper surfaces, resistance semicircles could be clearly visible in the high frequency range. Among them, that of copper modified by 1DT@PDA shows the greatest one. Bode and phase angle plots are also shown in Fig. 9b and c. 1-DT@PDA@Cu shows the maximum phase angle and impedance value. In contrast, the phase angle of PDA@Cu has a similar value to that of the bare copper but a relatively smaller impedance value, further confirming the presence of cracks in the PDA film.

To better understand the mechanism of the corrosion processes, the electrical equivalent circuit (EEC) models were fitted for the EIS data by using ZsimpWin software as demonstrated in Fig. 10. In detail, the model of $R(Q(R W))$ is suitable for the bare copper, while $R(Q(R W))(C R)$ is better for the modified copper surfaces. The merits of fitting can be assessed by standard deviations $\left(\chi^{2}\right)$. In all cases, $\chi^{2}$ is in the range of $10^{-4}$ to $10^{-3}$, an indication of the reasonable simulations. Moreover, another reliable indication of the electrochemical interface in terms of both charge transfer and transport processes within the passive film is that the error for each simulated electrical element in the suggested model should be less than $10 \% \cdot{ }^{44}$ In the equivalent circuits of $\mathrm{Cu}$ modified with coatings, $R_{\mathrm{S}}$ represents the solution resistance, a time constant involving $R_{\mathrm{ct}}$, and $C_{\mathrm{dl}}$ is associated with the charge-transfer process occurring at the copper electrode-solution interface. $R_{\mathrm{ct}}$ is the chargetransfer resistance and $C_{\mathrm{dl}}$ is the constant phase element (CPE or $Q$ ) modeling the capacitance of the double-charge layer. The time constant $\left(Q_{\mathrm{f}}\left(R_{\mathrm{f}} W\right)\right)$ is proposed to consider the diffusion process, including the semi-infinite diffusion induced by corrosive reactants or corrosion products (described by the $W$ element). $R_{\mathrm{f}}$ is the resistance of the inhibitor-adsorption layer and $Q_{\mathrm{f}}$ is the CPE of the layer. The CPE can be described by the following equation: ${ }^{45}$

Table 4 Electrochemical parameters calculated from EIS measurements for different samples in 3 wt $\% \mathrm{NaCl}$ aqueous solution

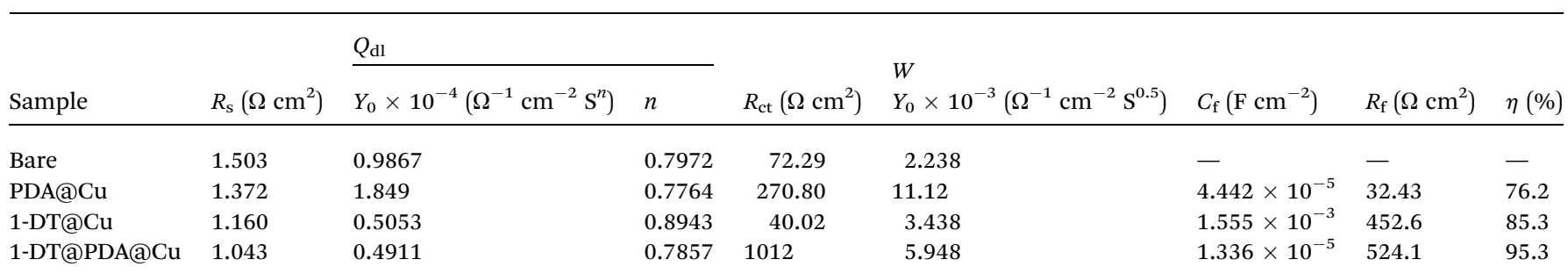




$$
Z_{\mathrm{CPE}}=Y_{0}^{-1}(\mathrm{j} \omega)^{-n}
$$

where $Y$ is the magnitude of CPE, $\mathrm{j}$ is the imaginary number, $\omega=2 \pi f$ represents the angular frequency $(f$ is the frequency in $\mathrm{Hz}$ ) and $n$ is the CPE exponent in relation with the details about the degree of surface inhomogeneity with respect to surface roughness, inhibitor adsorption, porous layer formation, etc. The smaller the $n$, the rougher the surface morphology of the electrode, due to serious electrode corrosion. ${ }^{46}$ Typically, for $n=$ $0, Z_{\mathrm{CPE}}$ is a resistance, when $n=1$, a capacitance, and for $n=$ -1 , an inductance. In general, for an electrode in a real scenario, the $n$ value ranges from 0 to 1.1-DT@PDA@Cu has the largest value of $n$ (0.8943). The fitted electrochemical parameters are tabulated in Table 4 . It is obvious that $R_{\mathrm{ct}}\left(270.80 \Omega \mathrm{cm}^{2}\right)$ of PDA@Cu is higher than that of the bare copper $\left(72.29 \Omega \mathrm{cm}^{2}\right)$. After modification with 1-DT, $R_{\mathrm{ct}}$ of 1-DT@PDA@Cu sharply increases to $1012 \Omega \mathrm{cm}^{2}$, indicating the improvement of inhibition. On the other hand, the increase of the $R_{\mathrm{f}}$ value also indicates the increase of the protection efficiency of the coating. As a result, the largest value of the 1-DT@PDA film resistance $\left(R_{\mathrm{f}}\right)$ consequently reveals that 1-DT in the PDA film cracks increases the hydrophobicity.

In addition, the inhibition efficiency of the coatings can be obtained using the following equation:

$$
\eta \%=\frac{R_{\mathrm{p}}-R_{\mathrm{p}}^{0}}{R_{\mathrm{p}}} \times 100 \%
$$
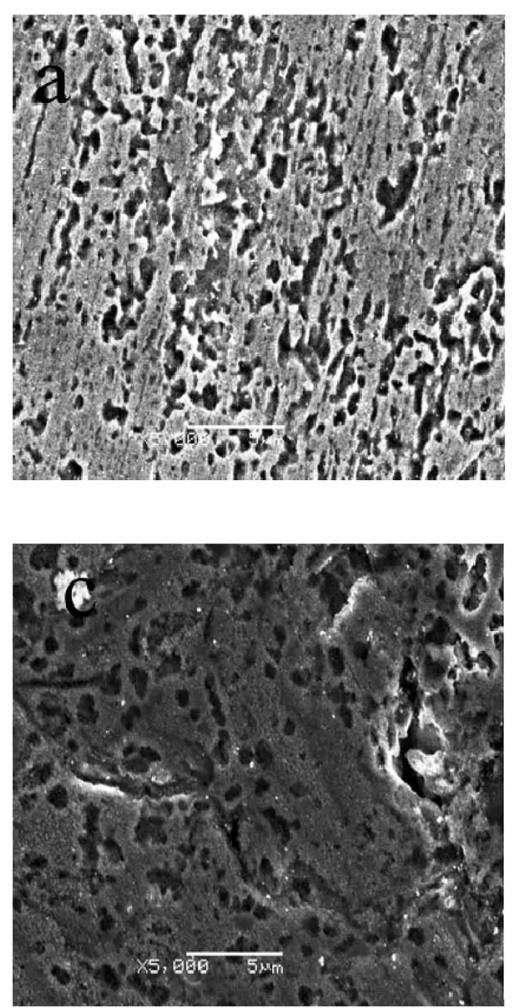

$R_{\mathrm{p}}^{0}$ is the resistance of the bare copper and $R_{\mathrm{p}}$ is the total resistance of the electrode with surface modification. Clearly, neglecting the solution resistance, which is relatively small and almost constant in all cases, the total resistance is the sum of $R_{\mathrm{ct}}$ and $R_{\mathrm{f}} \cdot{ }^{47,48}$ As expected, a high inhibition efficiency of $95.3 \%$ can be reached for 1-DT@PDA@Cu in $\mathrm{NaCl}$ aqueous solution. The EIS observations are in good accordance with the results from the polarization measurements.

It should be said that the inhibition of corrosion of 1DT@Cu, obtained from OCP results, seems to be different from those obtained from EIS and electrochemical polarization experiments. Hence, we repeated the OCP experiments for PDA@Cu and 1-DT@Cu several times. For PDA@Cu, as shown in Fig. $\mathrm{S} 1$ (ESI $\dagger$ ), the OCP results recorded three times are in quite good agreement, while for 1-DT@Cu, the OCP results are different (see Fig. S2 in ESI $\dagger$ ). The possible reasons are that the OCP method is a slow dynamic process and the 1-DT monolayer might be undergoing re-organization, which results in various structures of the layers, poor uniformity and density and OCP variation, but the PDA coatings are thick enough to provide stable OCPs. In contrast, EIS and electrochemical polarization are quick processes, which could indicate the behavior of the original 1-DT monolayer at the copper surface.

\subsection{SEM observations}

Fig. 11 shows SEM images of the bare copper and modified copper substrates, which have been immersed in $3 \mathrm{wt} \% \mathrm{NaCl}$ aqueous solution for $3 \mathrm{~h}$. Obviously, the localized corrosion
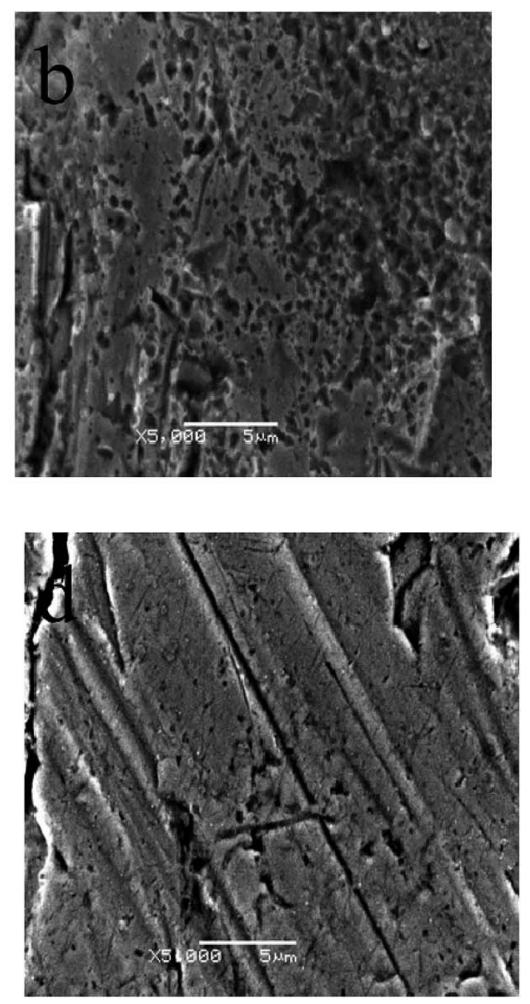

Fig. 11 SEM images of copper surfaces: (a) bare Cu, (b) PDA@Cu, (c) 1-DT@Cu, and (d) 1-DT@PDA@Cu, directly immersed in 3 wt\% NaCl aqueous solution for $3 \mathrm{~h}$ (corrosion time) at $298 \mathrm{~K}$. 
occurred extensively on the bare copper. With the modification with only PDA or 1-DT, the degree of corrosion decreases. $\mathrm{Cu}$ with the 1-DT@PDA coating seems smooth and only some tiny inevitable pitting corrosion occurs (compare to the bare copper in Fig. 11a) after immersion in corrosive media. Therefore, hydrophobic 1-DT@PDA layers result in improved protection of the copper surface from salt attack.

\section{Conclusions}

In summary, by a facile protocol, a 1-DT@PDA coating at the copper surface was fabricated. The resulting film showed high quality hydrophobicity with a contact angle of $110^{\circ}$, whose inhibition efficiency reached $95.3 \%$ through an electrochemical method. FTIR, NIR and XPS results elucidated that the greatest protection capability of 1-DT@PDA for copper from corrosion in $3 \mathrm{wt} \% \mathrm{NaCl}$ aqueous solution should be due to the synergy effect of a uniform PDA coating and 1-DT in the cracks of PDA directly interacting with the copper surface via the formation of $\mathrm{Cu}-\mathrm{S}$ bonds. SEM observation also exhibited the well-protected surface of 1-DT@PDA@Cu.

\section{Acknowledgements}

This work is supported by the National Natural Science Foundation of China (No. 21475088), International Joint Laboratory on Resource Chemistry (IJLRC), Shanghai Key Laboratory of Rare Earth Functional Materials, and Shanghai Municipal Education Committee Key Laboratory of Molecular Imaging Probes and Sensors.

\section{References}

1 H. Tian, W. Li, K. Cao and B. Hou, Potent inhibition of copper corrosion in neutral chloride media by novel nontoxic thiadiazole derivatives, Corros. Sci., 2013, 73, 281-291.

2 F. H. Su and K. Yao, Facile fabrication of superhydrophobic surface with excellent mechanical abrasion and corrosion resistance on copper substrate by a novel method, ACS Appl. Mater. Interfaces, 2014, 6, 8762-8770.

3 W. Liu, Q. J. Xu, J. Han, X. H. Chen and Y. L. Min, A novel combination approach for the preparation of superhydrophobic surface on copper and the consequent corrosion resistance, Corros. Sci., 2016, 110, 105-113.

$4 \mathrm{M}$. Mousavi and T. Baghgoli, Application of interaction energy in quantitative structure-inhibition relationship study of some benzenethiol derivatives on copper corrosion, Corros. Sci., 2016, 105, 170-176.

5 G. Zerjav and I. Milosev, Protection of copper against corrosion in simulated urban rain by the combined action of benzotriazole, 2-mercaptobenzimidazole and steric acid, Corros. Sci., 2015, 98, 180-191.

6 A. Drach, I. Tsukrov, J. Decew, J. Aufrecht, A. Grobauer and U. Hofmannn, Field studies of corrosion behaviour of copper alloys in natural seawater, Corros. Sci., 2013, 76, 453-464.
7 T. Tuken, N. Kicir, N. T. Elalan, G. Sigircik and M. Erbil, Self assembled film based on hexane-1,6-diamine and 2mercapto-ethanol on copper, Appl. Surf. Sci., 2012, 258, 6793-6799.

$8 \mathrm{~K}$. F. Khaled, Corrosion control of copper in nitric acid solutions using some amino acids-a combined experimental and theoretical study, Corros. Sci., 2010, 52, 3225-3234.

9 K. C. Chang, H. I. Lu, C. W. Peng, M. C. Lai, S. C. Hsu, M. H. Hsu, Y. K. Tsai, C. H. Chang, W. I. Hung, Y. Wei and J. M. Yeh, Nanocasting technique to prepare lotus-leaf-like superhydrophobic electroactive polyimide as advanced anticorrosive coatings, ACS Appl. Mater. Interfaces, 2013, 5, 1460-1467.

10 T. Kamegawa, Y. Shimizu and H. Yamashita, Superhydrophobic surfaces with photocatalytic selfcleaning properties by nanocomposite coating of $\mathrm{TiO}_{2}$ and polytetrafluoroethylene, Adv. Mater., 2012, 24, 3697-3700.

11 B. Su, S. T. Wang, Y. C. Wu, X. Chen, Y. L. Song and L. Jiang, Small molecular nanowire arrays assisted by superhydrophobic pillar-structured surfaces with high adhesion, Adv. Mater., 2012, 24, 2780-2785.

12 P. Wang, D. Zhang, R. Qiu and J. J. Wu, Super-hydrophobic metal-complex film fabricated electrochemically on copper as a barrier to corrosive medium, Corros. Sci., 2014, 83, 317-326.

13 P. Wang, D. Zhang, R. Qiu, Y. Wan and J. J. Wu, Green approach to fabrication of a super-hydrophobic film on copper and the consequent corrosion resistance, Corros. Sci., 2014, 80, 366-373.

14 R. Qiu, D. Zhang and P. Wang, Superhydrophobic-carbon fibre growth on a zinc surface for corrosion inhibition, Corros. Sci., 2013, 66, 350-359.

15 L. J. Li, Y. Z. Zhang, J. L. Lei, J. X. He, R. Lv, N. B. Li and F. S. Pan, A facile approach to fabricate superhydrophobic $\mathrm{Zn}$ surface and its effect on corrosion resistance, Corros. Sci., 2014, 85, 171-182.

16 J. Y. Zhang and Z. X. Kang, Effect of different liquid-solid contact models on the corrosion resistance of superhydrophobic magnesium surfaces, Corros. Sci., 2014, 87, 452-459.

17 H. Y. Ma, C. Yang, S. H. Chen, Y. L. Jiao, S. X. Huang, D. G. Li and J. L. Luo, Electrochemical investigation of dynamic interfacial processes at 1-octadecanethiol-modified copper electrodes in halide-containing solutions, Electrochim. Acta, 2003, 48, 4277-4289.

18 Z. Petrovic, M. Metikos-Hukovic and R. Babic, Modification of copper with self-assembled organic coatings, Prog. Org. Coat., 2008, 61, 1-6.

19 B. Lussem, L. Muller-Meskamp, S. Karthauser and R. Waser, A new phase of the $c(4 \times 2)$ superstructure of alkanethiols grown by vapor phase deposition on gold,, Langmuir, 2005, 21, 5256-5258.

20 A. H. David and C. Q. Liu, Oxidation protection of copper surfaces using self-assembled monolayers of octadecanethiol, Appl. Surf. Sci., 2005, 252, 400-411. 
21 Z. Ghelichkhha, S. Sharifi-Asl, K. Farhadi, S. Banisaied, S. Ahmadi and D. D. Macdonald, L-Cysteine/polydopamine nanoparticle-coatings for copper corrosion protection, Corros. Sci., 2015, 91, 129-139.

22 F. S. Pan, H. P. Ji, S. Z. Qiao, Z. Y. Jiang, J. T. Wang, B. Y. Wang and Y. R. Zhong, Bioinspired fabrication of high performance composite membranes with ultrathin defect-free skin layer, J. Membr. Sci., 2009, 341, 279-285.

23 B. Li, W. P. Liu, Z. Y. Jiang, X. Dong, B. Y. Wang and Y. R. Zhong, Ultrathin and stable active layer of dense composite membrane enabled by poly(dopamine), Langmuir, 2009, 25, 7368-7374.

24 J. L. Dalsin and P. B. Messersmith, Bioinspired antifouling polymers, Mater. Today, 2005, 8, 38-46.

25 S. Haemers, G. J. M. Koper and G. Frens, Effect of oxidation rate on cross-linking of mussel adhesive proteins, Biomacromolecules, 2003, 4, 632-640.

26 L. A. Burzio and J. H. Waite, Cross-linking in adhesive quinoproteins: studies with model decapeptides, Biochemistry, 2000, 39, 11147-11153.

$27 \mathrm{~J}$. Monahan and J. J. Wilker, Cross-linking the protein precursor of marine mussel adhesives: bulk measurements and reagents for curing, Langmuir, 2004, 20, 3724-3729.

28 W. Zhang, F. K. Yang, Y. G. Han, R. Gaikwad, Z. Leonenko and B. Zhao, Surface and tribological behaviors of the bioinspired polydopamine thin films under dry and wet conditions, Biomacromolecules, 2013, 14, 394-405.

29 B. P. Lee, J. L. Dalsin and P. B. Messersmith, Synthesis and gelation of DOPA-modified poly(ethylene glycol) hydrogels, Biomacromolecules, 2002, 3, 1038-1047.

30 M. J. LaVoie, B. L. Ostaszewski, A. Weihofen, M. G. Schlossmacher and D. J. Selkoe, Dopamine covalently modifies and functionally inactivates parkin, Nat. Med., 2005, 11, 1214-1221.

31 S. Devillers, A. Hennart, J. Delhalle and Z. Mekhalif, 1Dodecanethiol self-assembled monolayers on cobalt, Langmuir, 2011, 27, 14849-14860.

32 N. Sandhyarani and T. Pradeep, Current understanding of the structure, phasetransitions and dynamics of selfassembled monolayers on two- and three-dimensional surfaces, Int. Rev. Phys. Chem., 2003, 22, 221-262.

33 H. Wang, S. F. Chen, L. Y. Li and S. Y. Jiang, Improved method for the preparation of carboxylic acid andamine terminated self-assembled monolayers of alkanethiolates, Langmuir, 2005, 21, 2633-2636.

34 M. L. Wallwork, D. A. Smith, J. Zhang, J. Kirkham and C. Robinson, Complex chemical force titration behavior of amine-terminated self-assembled monolayers, Langmuir, 2001, 17, 1126-1131.

35 F. Sinapi, S. Julien, D. Auguste, L. Hevesi, J. Delhalle and Z. Mekhalif, Monolayers and mixed-layers on copper towards corrosion protection, Electrochim. Acta, 2008, 53, 4228-4238.
36 Z. Mekhalif, F. Sinapi, F. Laffineur and J. Delhalle, XPS and electrochemical characterisation of polycrystalline copper modified with 12-( $N$-pyrrolyl)- $n$-dodecanethiol, $J$. Electron Spectrosc. Relat. Phenom., 2001, 201, 149-161.

37 P. Wang, C. H. Liang, B. Wu, N. B. Huang and J. L. Li, Protection of copper corrosion by modification of dodecanethiol self-assembled monolayers prepared in aqueous micellar solution, Electrochim. Acta, 2010, 55, 878883.

38 J. H. Jiang, L. P. Zhu, L. J. Zhu, H. T. Zhang, B. K. Zhu and Y. Y. Xu, Antifouling and antimicrobial polymer membranes based on bioinspired polydopamine and strong hydrogen-bonded poly( $N$-vinyl pyrrolidone), ACS Appl. Mater. Interfaces, 2013, 5, 12895-12904.

39 Y. Q. Chen, S. Zhao, M. Y. Chen, W. T. Zhang, J. L. Mao and Y. C. Zhao, Sandwiched polydopamine (PDA) layer for titanium dioxide $\left(\mathrm{TiO}_{2}\right)$ coating on magnesium to enhance corrosion protection, Corros. Sci., 2015, 96, 67-73.

40 F. Yu, S. G. Chen, Y. Chen, H. M. Li, L. J. Yang, Y. Y. Chen and Y. S. Yin, Experimental and theoretical analysis of polymerization reaction process on the polydopamine membranes and its corrosion protection properties for 304 stainless steel, J. Mol. Struct., 2010, 982, 152-161.

41 T. P. Ang and W. S. Chin, Dodecanethiol-Protected copper/ silver bimetallic nanoclusters and their surface properties, J. Phys. Chem. B, 2005, 109, 22228-22236.

42 N. T. Kirkland, N. Birbilis and M. P. Staiger, Assessing the corrosion of biodegradable magnesium implant: a critical review of current methodologies and their limitations, Acta Biomater., 2012, 8, 925-936.

43 T. H. Hu, H. W. Shi, T. Wei, F. C. Liu, S. H. Fan and E. H. Han, Cerium tartrate as a corrosion inhibitor for AA 2024-T3, Corros. Sci., 2015, 95, 152-161.

44 H. W. Tian, Y. F. Cheng, W. H. Li and B. R. Hou, Triazolylacylhydrazone derivatives as novel inhibitors for copper corrosion in chloride solutions, Corros. Sci., 2015, 100, 341-352.

45 G. Brug, A. V. D. Eeden, M. Sluyters-Rehbach and J. H. Sluyters, The analysis of electrode impedances complicated by the presence of a constant phase element,, J. Electroanal. Chem., 1984, 176, 275-295.

46 K. F. Khaled and N. Hackerman, Ortho-substituted anilines to inhibit copper corrosion in aerated $0.5 \mathrm{M}$ hydrochloric acid, Electrochim. Acta, 2004, 49, 485-495.

47 J. T. Zhang, J. Zhao, N. S. Zhang, C. T. Qu and X. Zhang, Synergized action of $\mathrm{CuCl}$ on recycled cigarette butts as corrosion inhibitor for $\mathrm{N} 80$ steel at $90{ }^{\circ} \mathrm{C}$ in $15 \% \mathrm{HCl}$, Ind. Eng. Chem. Res., 2011, 50, 7264-7272.

48 K. F. Khaled, Experimental and atomistic simulation studies of corrosion inhibition of copper by a new benzotriazole derivative in acid medium, Electrochim. Acta, 2009, 54, 4345-4352. 